\title{
Attosecond sampling of arbitrary optical waveforms
}

\author{
Adam S. Wyatt,,${ }^{1,2}$ Tobias Witting, ${ }^{3}$ Andrea Schiavi, ${ }^{1}$ Davide Fabris, ${ }^{3}$ Paloma Matia-Hernando, ${ }^{3}$ \\ Ian A. Walmsley, ${ }^{1}$ Jon P. Marangos, ${ }^{3}$ and John W. G. Tisch ${ }^{3, *}$ \\ ${ }^{1}$ Clarendon Laboratory, Department of Physics, University of Oxford, Oxford OX1 3PU, UK \\ ${ }^{2}$ Central Laser Facility, STFC Rutherford Appleton Laboratory, Harwell OX11 0QX, UK \\ ${ }^{3}$ Blackett Laboratory, Imperial College London, London SW7 2AZ, UK \\ ${ }^{*}$ Corresponding author: john.tisch@imperial.ac.uk
}

Received 10 December 2015; revised 2 February 2016; accepted 2 February 2016 (Doc. ID 255541); published 17 March 2016

\begin{abstract}
Advances in the generation of ultrashort laser pulses, and the emergence of new research areas such as attosecond science, nanoplasmonics, coherent control, and multidimensional spectroscopy, have led to the need for a new class of ultrafast metrology that can measure the electric field of complex optical waveforms spanning the ultraviolet to the infrared. Important examples of such waveforms are those produced by spectral control of ultrabroad bandwidth pulses, or by Fourier synthesis. These are typically tailored for specific purposes, such as to increase the photon energy and flux of high-harmonic radiation, or to control dynamical processes by steering electron dynamics on subcycle time scales. These applications demand a knowledge of the full temporal evolution of the field. Conventional pulse measurement techniques that provide estimates of the relative temporal or spectral phase are unsuited to measure such waveforms. Here we experimentally demonstrate a new, all-optical method for directly measuring the electric field of arbitrary ultrafast optical waveforms. Our method is based on high-harmonic generation (HHG) driven by a field that is the collinear superposition of the waveform to be measured with a stronger probe laser pulse. As the delay between the pulses is varied, we show that the field of the unknown waveform is mapped to energy shifts in the high-harmonic spectrum, allowing a direct, accurate, and rapid retrieval of the electric field with subcycle temporal resolution at the location of the HHG.
\end{abstract}

Published by The Optical Society under the terms of the Creative Commons Attribution 4.0 License. Further distribution of this work must maintain attribution to the author(s) and the published article's title, journal citation, and DOI.

OCIS codes: (320.0320) Ultrafast optics; (320.7100) Ultrafast measurements; (140.0140) Lasers and laser optics; (190.2620) Harmonic generation and mixing.

http://dx.doi.org/10.1364/OPTICA.3.000303

\section{INTRODUCTION}

Ultrashort duration optical pulses find application in a wide range of science and technology, from measurement and control of molecular and electronic dynamics [1-5], to material processing $[6,7]$. Knowledge of the pulse shape - that is, the temporal (and possibly spatial) distribution of the pulse's electric field-is important both as a diagnostic of the laser sources producing the pulses, and also for understanding the outcomes of experiments or applications that use them. In the past decade, the development of light pulses in which the phase of the carrier frequency relative to the envelope is controllable $[8,9]$ have made it necessary to consider methods that can retrieve the full electric field, rather than simply the field envelope. Despite considerable success in the development of schemes for pulse characterization [10], it has proven challenging to find schemes that can extract the entire temporal field. The reason for this is that the methods are selfreferencing, meaning that they use the pulse to "measure" itself. This necessarily means that they are insensitive to time-invariant parameters of the field. The well-established methods of the past two decades, including FROG [11], SPIDER [12], MIIPS [13], and D-Scan [14], have played important roles in the development of ultrafast laser sources and for characterizing laser pulses used in a wide range of experiments. However, they suffer from a number of limitations in their capabilities: (i) they measure only the spectral/temporal intensity and dispersion/chirp, not the full electric field, including the carrier-envelope phase (CEP); (ii) they cannot measure the relative phase across spectral nulls without an auxiliary field with a bandwidth that spans the null $[15,16]$; (iii) for ultrabroad bandwidth pulses, the bandwidth and efficiency of the measurement is limited by the requirement to use a nonlinear material to act as a time-stationary filter; and (iv) the pulse is rarely measured in situ, which necessitates a careful calibration of the linear and nonlinear space-time propagation of the pulse from the diagnostic to the site of the experiment.

Developments in the synthesis of complex pulse waveforms have pushed the metrology requirements beyond the capabilities of these conventional characterization techniques, providing a motivation for new methods suited to the measurement of arbitrary waveforms. Complex waveforms in the ultraviolet (UV) to 
infrared spectral regions have been obtained by a variety of methods, including spectral phase control of ultrawide bandwidth pulses $[17,18]$, or by the Fourier synthesis of discrete harmonics [19]. Such waveforms are often tailored for field-sensitive lightmatter studies. Examples include the generation of subcycle transients for attosecond spectroscopy in the condensed phase [20], and the synthesis of sawtooth waveforms to optimize high-harmonic generation (HHG) [21,22] or terahertz pulse generation [23]. Accurate and robust ways of measuring such waveforms with subcycle temporal resolution are required to support future developments in ultrafast and attosecond science. What is needed is a reference field and a medium with a very rapid nonlinear response, significantly shorter than an optical cycle. The process of HHG provides such a mechanism. Ideally, the measurement technique should also allow sufficiently rapid acquisition of data (of the order of seconds) to permit near-real-time monitoring of waveforms.

To the best of our knowledge, there are currently only two techniques that have been demonstrated to retrieve the electric field of an arbitrary optical waveform. The first is attosecond streaking [24-26], in which photoelectrons produced by an attosecond pulse [usually in the extreme ultraviolet (XUV)] are momentum-shifted (streaked) by the field of the pulsed waveform to be measured. Measuring this momentum shift as a function of delay between the pulses allows the unknown waveform to be retrieved. Attosecond streaking has been used mostly to characterize attosecond pulses. However, it has also been applied to characterize near-infrared (NIR) few-cycle pulses to allow comparison with other techniques, such as SPIDER [27], and has recently been used to measure synthesized waveforms spanning three octaves from the UV to NIR [28]. For $1 \mathrm{kHz}$ repetition lasers, minimum scan times are of the order of minutes and, more typically, tens of minutes.

A second method has recently been reported by Kim et al. [29]. In their all-optical approach, the pulse to be measured is crossed at an angle with an intense drive pulse for HHG, resulting in a deflection of the XUV harmonic pulse within a macroscopic sample by an amount proportional to the test pulse electric field at the time of overlap. The waveform of the test pulse can thus be retrieved by measuring this deflection as a function of delay between the pulses using a spatially resolving XUV spectrometer. This all-optical method removes the complexities of attosecond pulse generation and photoelectron spectroscopy that are required for attosecond streaking.

Here, we report on a new all-optical HHG-based method that has some important differences to the technique of Kim et al. It uses collinear beams, does not require a spatially resolving spectrometer, and it uses only the spectral amplitude manifested in the HHG intensity spectrum. These features allow for a straightforward and versatile implementation and very rapid and direct field retrieval. Our technique, called Attosecond Resolved Interferometric Electric-Field Sampling (ARIES), is based on HHG driven by a field that is the collinear, parallel polarized superposition of the pulse to be measured, the test pulse (TP), and a more intense HHG drive pulse, the probe pulse (PP). As the delay between the pulses is varied, the field experienced by the atoms increases or decreases as the PP and TP fields add constructively or destructively. Therefore, the photon energy of the HHG cutoff, which is directly related to the incident peak intensity, changes. By monitoring the changes of the harmonic cut-off frequency as a function of the delay between the PP and TP, the full TP electric-field waveform can be extracted. In this way, the field of the TP is mapped to photon energy shifts in the high-harmonic spectrum that can be measured with a spectrometer. Using pulses centered at $800 \mathrm{~nm}$, we demonstrate the field sensitivity of ARIES by measuring the CEP changes of a fewcycle TP, and show further that ARIES can accurately retrieve the electric field of heavily chirped TPs. We also demonstrate the use of ARIES to measure the electric field of a UV TP with a center wavelength of $\approx 400 \mathrm{~nm}$. To the best of our knowledge, this is the highest frequency electric-field waveform that has been directly measured using all-optical means.

\section{ARIES WORKING PRINCIPLE}

ARIES works by mapping the TP waveform onto modulations in the HHG spectrum. Here we use modulations of the HHG spectral cutoff (highest photon energy) driven by a few-cycle PP as an easily tracked feature in the HHG spectrum. The HHG driving field in an ARIES measurement is given by $E(t)=E_{\mathrm{pp}}(t)+$ $E_{\mathrm{tp}}(t-\tau)$, where $E_{\mathrm{pp}}(t)$ is the strong CEP-stable PP, $E_{\mathrm{tp}}(t)$ is the weaker (typically 100-1000 times less intense) TP to be measured, and $\tau$ is the relative delay. As illustrated in Fig. 1, the addition of the TP shifts the cutoff compared to that which would be achieved by the PP alone. Constructive interference between the PP and TP during the generation of the cut-off emission (around the peak of the PP) shifts the cutoff to higher photon energies [Figs. 1(c) and 1(d)]. Destructive interference shifts the cutoff to lower energies [Figs. 1(e) and 1(f)]. By scanning the delay between the PP and TP, the TP waveform is mapped onto modulations in the HHG cutoff [Fig. 1(g)].

Within the framework of the semiclassical recollision model of HHG [30], the electron is ionized by the intense laser field, and then driven by the linearly polarized field away from and then back toward its parent ion. During recollision with the parent ion, the electron can recombine, leading to the emission of a photon of energy equal to the sum of its kinetic energy upon recollision and the ionization potential, $I_{p}$. The classical electron velocity at the instant of recollision for the two-component driving field is

$$
v=-\frac{e}{m} \int_{t_{i}}^{t_{r}}\left(E_{\mathrm{pp}}(t)+E_{\mathrm{tp}}(t-\tau)\right) \mathrm{d} t=v_{\mathrm{pp}}+v_{\mathrm{tp}},
$$

where $e$ and $m$ are the electron charge and mass, respectively, and $t_{i}$ and $t_{r}$ are the ionization and recollision times, respectively. Writing $E_{\mathrm{pp}}=\hat{E}_{\mathrm{pp}} \cos \omega_{\mathrm{pp}} t$ and $E_{\mathrm{tp}}=\hat{E}_{\mathrm{tp}} \cos \left(\omega_{\mathrm{tp}} t+\phi\right)$, where $\hat{E}_{\mathrm{pp}}, \hat{E}_{\mathrm{tp}}$, and $\omega_{\mathrm{pp}}, \omega_{\mathrm{tp}}$ are the amplitudes and frequencies of the PP and TP, respectively, we have from Eq. (1) that $v_{\mathrm{pp}} \propto \hat{E}_{\mathrm{pp}} / \omega_{\mathrm{pp}}$ and $v_{\text {tp }} \propto \hat{E}_{\text {tp }} / \omega_{\text {tp. }}$. The electron recollision kinetic energy is given by

$$
\varepsilon=\frac{1}{2} m\left(v_{\mathrm{pp}}+v_{\mathrm{tp}}\right)^{2} \approx \frac{1}{2} m v_{\mathrm{pp}}^{2}+m v_{\mathrm{pp}} v_{\mathrm{tp}},
$$

where we have neglected the term $\frac{1}{2} m v_{\text {tp }}^{2}$ since $\hat{E}_{\text {tp }} \ll \hat{E}_{\mathrm{pp}}$. If we consider the PP in isolation, it is well known that there is a particular electron trajectory with $t_{i}=t_{i, \mathrm{pp}}^{(\max )}$ and $t_{r}=t_{r, \mathrm{pP}}^{(\max )}$ that maximizes the recollision kinetic energy $\left(=\varepsilon_{\mathrm{pp}}^{(\max )}\right)$, corresponding to the cut-off HHG emission for the PP alone at a photon energy $I_{p}+\varepsilon_{\mathrm{pp}}^{(\max )}$. The addition of a much weaker TP changes these times slightly, but if we make the simplifying assumption 

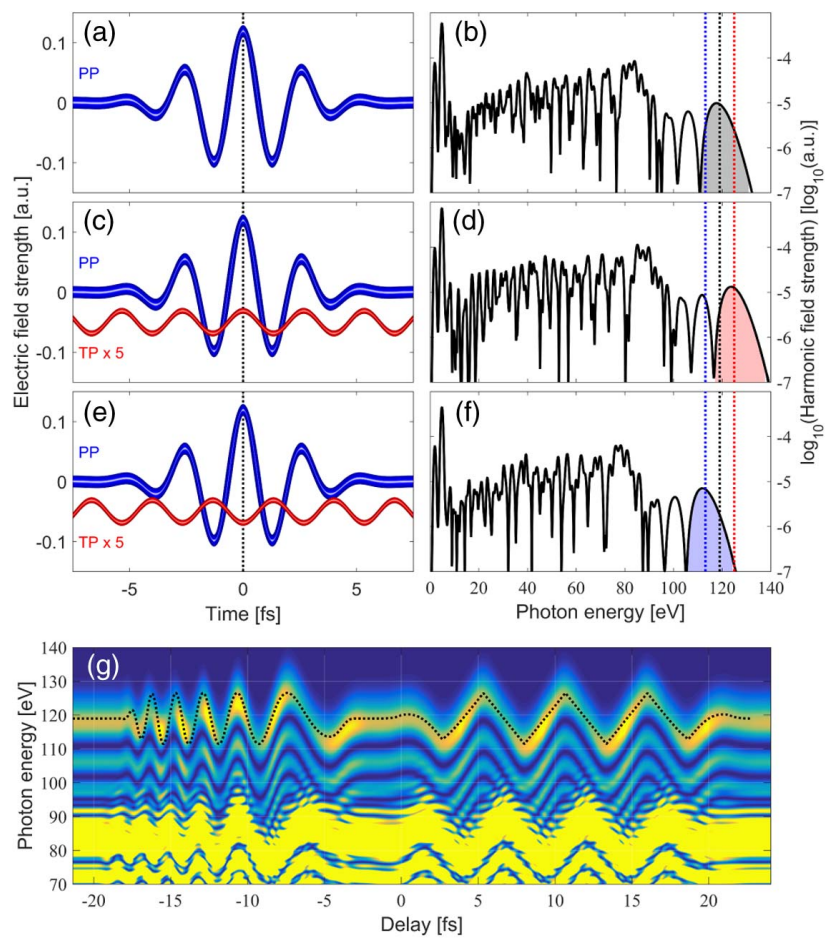

Fig. 1. Basic working principle of ARIES, demonstrated here by SFA calculations of HHG in Ne for a 3.5 fs probe pulse centered at a wavelength of $800 \mathrm{~nm}$ with a peak intensity of $5 \times 10^{14} \mathrm{~W} \mathrm{~cm}^{-2}$, and a continuous-wave $(\mathrm{CW})$ test waveform at $800 \mathrm{~nm}$ with a relative intensity $I_{\text {tp }} / I_{\mathrm{pp}}=0.1 \%$. (a) CEP-stabilized few-cycle probe pulse and (b) corresponding HHG spectrum. (c) Constructive interference with a CW test waveform and (d) resulting HHG spectrum. (e) Destructive interference with a CW test waveform and (f) resulting HHG spectrum. The same probe pulse (blue) was used in (a)-(f); the test waveform (red) in (c) and (e) has been magnified by a factor of $5 \times$ for clarity. In (b), (d), and (f), the spectral positions of the cutoff (dotted vertical lines) are calculated by using the corresponding shaded regions (black, probe only; red, constructive interference; blue, destructive interference). (g) Simulated ARIES scan for a complex test waveform that comprises a linearly chirped section with a frequency sweep from $\omega_{\text {tp }}=0.05 \omega_{\mathrm{pp}}-1.0 \omega_{\mathrm{pp}}$ and a sawtooth section of frequency $\omega_{\mathrm{tp}}=0.5 \omega_{\mathrm{pp}}$. Only the scan near the cut-off region is shown. The HHG spectrum as a function of delay between the probe and test pulses shows modulations of the cut-off that accurately map the TP electric-field waveform (dotted black curve, scaled in amplitude to match the maximum cut-off shift).

that this is a small effect and use $t_{i}=t_{i, \mathrm{pp}}^{(\max )}$ and $t_{r}=t_{r, \mathrm{pP}}^{(\max )}$ in Eq. (2), we obtain

$$
\Delta \varepsilon_{\max } \approx m v_{\mathrm{pp}} v_{\mathrm{tp}} \propto \frac{\hat{E}_{\mathrm{pp}} \hat{E}_{\mathrm{tp}}}{\omega_{\mathrm{pp}} \omega_{\mathrm{tp}}},
$$

where $\Delta \varepsilon_{\max }$ is the shift of the cutoff relative to $\varepsilon_{\mathrm{pp}}^{(\max )}$. The heterodyne mixing responsible for the cross-term $m v_{\text {pp }} v_{\text {tp }}$ has been considered theoretically in the context of extending the HHG cutoff by using a weak control pulse [31]. In that work, the linear dependence of the cut-off shift on the electric field of the weaker field (here $\hat{E}_{\text {tp }}$ ) obtained from the semiclassical analysis [Eq. (3)] was confirmed via simulations of HHG by numerical integration of the time-dependent Schrödinger equation using pulsed fields. The ARIES method utilizes this linear dependence to retrieve the TP electric-field waveform by recording $\Delta \varepsilon_{\max }(\tau)$, i.e., the cut-off shift as a function of the delay between the PP and TP. The temporal resolution of the measurements is related to the time interval $\Delta t=t_{r}-t_{i}$. Under the simplifying assumption that $\Delta t$ is independent of $\tau, \Delta \varepsilon_{\max }(\tau)$ is simply proportional to the convolution of $E_{\mathrm{tp}}(t)$ with a gate function of width $\Delta t$. Full numerical integration of Eq. (1) to find the cut-off electron trajectory in the combined field at each $\tau$ yields a full width at half-maximum temporal resolution of $<1 \mathrm{fs}$.

Going beyond these classical considerations, we can model the process more realistically by calculating the HHG emission from a single atom driven by the combined field, $E(t)=E_{\mathrm{pp}}(t)+$ $E_{\mathrm{tp}}(t-\tau)$, using the strong field approximation (SFA) [32], which is an analytic quantum theory. Simulated ARIES traces for a $3.5 \mathrm{fs} \mathrm{PP}$ at $800 \mathrm{~nm}$ and a TP waveform comprising a frequency chirped section and a sawtooth are shown in Fig. 1(g). The calculated HHG spectrum as a function of $\tau$ shows clear modulations in the cutoff that accurately retrieve the TP waveform.

\section{METHODS}

For our experimental demonstration of ARIES, we employed the setups shown in Figs. 2(a) and 2(b).

For measurements with an $800 \mathrm{~nm} \mathrm{PP}$ and TP [Fig. 2(a)], we used pulses from a few-cycle laser system (Femtolasers, Femtopower) comprising a CEP-stabilized Ti:sapphire femtosecond amplifier followed by a home-built differentially pumped hollow fiber and chirped mirror compressor [33,34]. A fused silica wedge pair was used to fine-tune the dispersion and CEP. At the output of this system, pulses of 3.5 fs duration with $0.5 \mathrm{~mJ}$ energy were obtained, at a repetition rate of $1 \mathrm{kHz}$. We use SEA-FSPIDER [35] to measure the few-cycle pulse durations. The CEP stability was better than 250 mrad RMS single-shot.

The test and probe pulses are produced by sending the few-cycle pulses into a Mach-Zehnder interferometer. The TP undergoes two reflections from broadband beam splitters of $10 \%$ reflectivity, while the probe pulse is transmitted by the beam splitters, giving a maximum intensity ratio $I_{\mathrm{tp}} / I_{\mathrm{pp}}$ of $\approx 1 \%$. We have also experimentally verified the ability to use a mirror with a hole in the center to split and recombine the beams instead of broadband beam splitters. This overcomes any limitations in obtaining beam splitters with suitable optical coatings and low dispersion from the substrate, allowing maximum flexibility in wavelength and bandwidth selection. An adjustable iris in the TP arm was used to adjust this ratio in the range of $0.1 \%$ $1 \%$. A fused silica wedge pair in the TP arm was used to control the TP CEP and chirp. By using the wedge pair, it was possible to balance the dispersion of the TP and PP. The relative dispersion and group delay were measured using spectral interferometry. Delay control in the interferometer was provided by a double delay stage comprising a closed-loop piezo actuated stage (Physik Instrumente, P-753 LISA) with $50 \mathrm{pm}$ resolution and $38 \mu \mathrm{m}$ range mounted on top of an open-loop DC motor stage (Thorlabs, PT1-Z8) with $200 \mathrm{~nm}$ resolution and $25 \mathrm{~mm}$ range. After the interferometer, both pulses entered a vacuum chamber through a $1 \mathrm{~mm}$ thick fused silica window. Inside the chamber, high-order harmonics were generated by focusing the collinear beams (both linearly polarized with parallel polarizations) with a $75 \mathrm{~cm}$ focal length spherical mirror into a neon gas target approximately $1 \mathrm{~mm}$ long with a density of $\approx 10^{17} \mathrm{~cm}^{-3}$. The high 
(a)

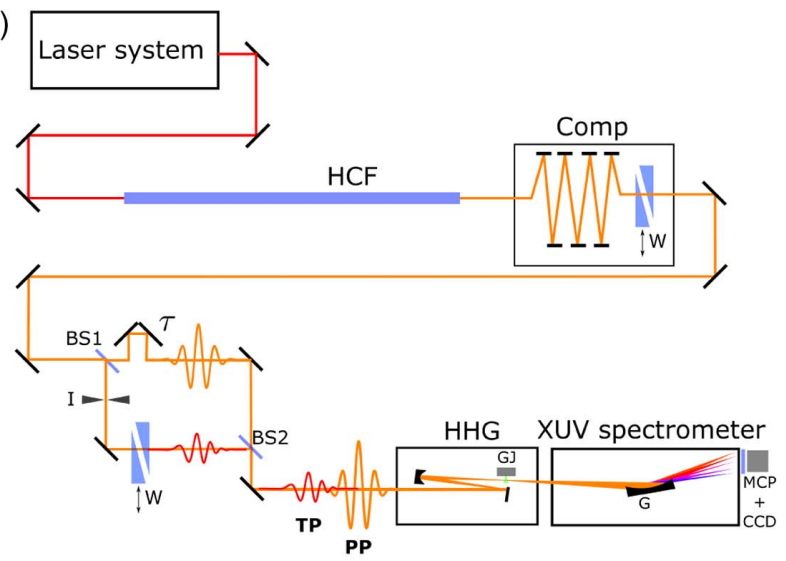

(b)

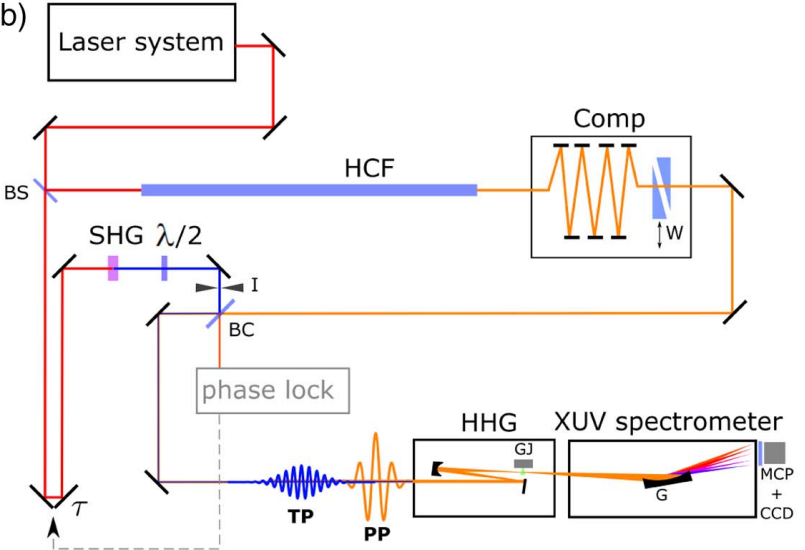

Fig. 2. Experimental apparatus used for demonstrating ARIES, (a) Setup used for electric-field measurements of $800 \mathrm{~nm}$ TPs. The PP and TP are produced in a Mach-Zehnder interferometer by injecting CEP-stable, few-cycle pulses from an amplified Ti:sapphire laser system with hollow core fiber (HCF) and chirped mirror compressor (Comp). Beam splitters BS1 and BS2 are 10\% reflective. The adjustable iris (I) in the TP arm is used to control the TP intensity, and the fused silica wedges (W) in the TP arm are used to control the TP CEP and chirp. A delay stage in the PP arm is used to control the relative delay, $\tau$, between the pulses. After the Mach-Zehnder interferometer, the collinear beams are sent into a HHG chamber, where they are focused into a gas jet (GJ). The generated harmonics are spectrally analyzed in an XUV spectrometer that uses a flat-field grating $(\mathrm{G})$. The HHG spectrum is detected on a microchannel plate detector (MCP) readout by a CCD camera. (b) Setup used for electric-field measurements of $400 \mathrm{~nm}$ TPs. A portion of the laser pulse is split off at beam splitter BS before the HCF, and used to generate a second-harmonic TP in a BBO SHG crystal. The polarization of the second-harmonic pulse is rotated by $90^{\circ}$ with a half-wave plate and combined with the few-cycle PP at a dichroic beam combiner (BC). Due to the long path length in this setup, the relative delay was actively stabilized.

harmonics were dispersed with a flat-field XUV spectrometer [36] and detected with a microchannel plate (MCP) detector (Photonis) read out with a cooled CCD camera (Photonic Science, Coolview FDI). This enabled HHG spectra to be recorded in the energy range 15-150 eV. Each harmonic spectrum was integrated over several hundred laser pulses.

For our ARIES measurements of a UV pulse, we used the setup shown in Fig. 2(b). A beam splitter was used to split off a $0.5 \mathrm{~mJ}, 30 \mathrm{fs}$ pulse before the hollow fiber. This was then frequency doubled in a $100 \mu \mathrm{m}$ thick Type I beta barium borate
(BBO) second-harmonic generation (SHG) crystal. The SHG pulse, centered at $\approx 400 \mathrm{~nm}$, was recombined with the $3.5 \mathrm{fs}$ $\mathrm{PP}$ at a dichroic beam combiner (Layertec). The collinear, parallel polarized beams were then directed to the HHG chamber, as for the $800 \mathrm{~nm}$ measurements. The PP parameters were as before. The TP intensity was $\approx 10^{11} \mathrm{Wcm}^{-2}\left(I_{\mathrm{tp}} / I_{\mathrm{pp}} \approx 0.05 \%\right)$.

For the ARIES field retrieval, the HHG cut-off energy is determined by calculating the center of mass of the spectrally masked on-axis harmonic emission spectrum. The on-axis emission is calculated by averaging over the central part of the spatially resolved harmonic spectrum defined by the region in which the harmonic spectrum depends weakly on the spatial position. This can also be achieved experimentally using a mask in the far field of the HHG source before reimaging onto a single array detector, eliminating the requirement to spatially resolve and thus reducing the acquisition time. The spectral mask is generated by finding the group of adjacent harmonic photon energies with a spectral density above a user-defined threshold located near the cut-off position of the PP-only spectrum.

\section{RESULTS AND DISCUSSION}

We begin with the results for the $800 \mathrm{~nm}$ TP. To test ARIES' sensitivity to the TP field, we performed several ARIES measurements as a function of TP CEP, while keeping the PP constant. The results are shown in Fig. 3.

The PP and TP both had a duration of $3.5 \mathrm{fs}$, with intensities $\approx 5 \times 10^{14} \mathrm{~W} \mathrm{~cm}^{-2}$ and $\approx 5 \times 10^{11} \mathrm{~W} \mathrm{~cm}^{-2}$, respectively,

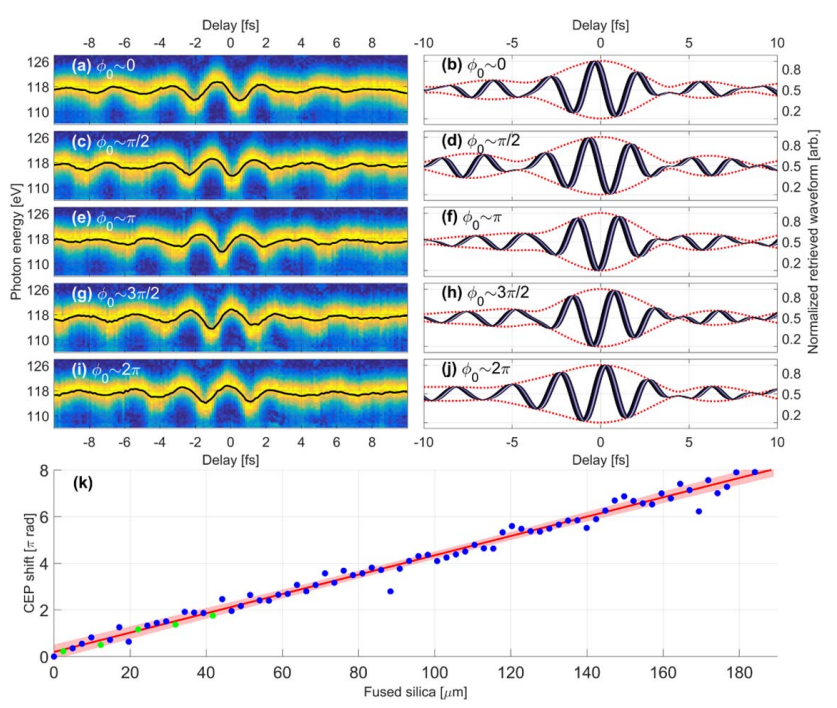

Fig. 3. ARIES measurements of few-cycle TP waveforms with varying CEP, demonstrating its field sensitivity. (a), (c), (e), (g), and (i) are measured ARIES scans for five different TP CEP values spanning a range of $\approx 2 \pi$. The black lines are the center of masses of the HHG cutoff as a function of delay. The corresponding field retrievals are replotted in (b), (d), (f), (h), and (j) as thick blue curves with the pulse envelopes plotted as dotted red lines. (k) Retrieved TP CEP values (blue circles) as a function of fused silica insertion obtained over a larger wedge scan range corresponding to $\approx 8 \pi \mathrm{CEP}$ phase variation. The solid line is a linear fit to the data points, with the shaded red region marking the $95 \%$ confidence interval. The CEP is defined as the mean wavelength times the temporal difference between the peak of the electric field and peak of the pulse envelope. The green points mark the wedge positions of the waveforms plotted in (b), (d), (f), (h), and (j); the retrieved CEP values are $0.2 \pi$, $0.5 \pi, 1.2 \pi, 1.4 \pi$, and $1.8 \pi$, respectively. 
$\left(I_{\mathrm{tp}} / I_{\mathrm{pp}} \approx 0.1 \%\right)$. Figures 3(a), 3(c), 3(e), 3(g), and 3(i) show ARIES scans for five different wedge positions, covering a CEP range of $\approx 2 \pi$. The black lines track the cut-off position as a function of delay. The delay-dependent shifts have been replotted as the thick curves in Figs. 3(b), 3(d), 3(f), 3(h), and 3(j). The CEP variation is clearly evident. We emphasize that, aside from finding the center of mass of the cut-off position at each delay (which is computationally straightforward), no additional processing is required to retrieve the normalized TP electric-field waveform. The retrieved CEP as a function of fused silica insertion for a larger range of wedge positions has been plotted in Fig. $3(\mathrm{k})$. The inverse slope of the linear fit to the data points is $48.5 \pm 0.7 \mu \mathrm{m} / 2 \pi \mathrm{rad}$, which is in excellent agreement with the theoretically predicted value of $49.1 \mu \mathrm{m} / 2 \pi \mathrm{rad}$ for fused silica.

We have also shown that ARIES can retrieve the field of significantly longer TPs that are heavily chirped (large timebandwidth product). To do this, we carried out a TP wedge scan over a much larger range, corresponding to around $1 \mathrm{~mm}$ of fused silica. The retrieved waveforms are shown in Fig. 4(a). Waveforms (I) and (II) are positively chirped, waveform (III) is close to the zero dispersion position, and waveforms (IV)-(VIII) are negatively chirped. Figure 4(c) show the average of waveforms (I)-(VIII) after numerically compensating each one for the known phase introduced by the wedges. The shaded region corresponds to the mean \pm 1 standard deviation, showing the precision of the retrieval even for heavily chirped TPs. Figure 4(b) shows the spectral phase of the retrieved pulses minus the spectral phase of the near-Fourier-limited pulse (III), together with the theoretical spectral phase based on the known fused silica insertion. The agreement between the retrieved and theoretical curves is excellent. In Fig. 4(d), the retrieved TP spectrum is compared to an independent measurement of the spectrum using SEA-FSPIDER. As in [29], the spectra agree qualitatively.

We now turn to our measurements of a UV pulse centered at $\approx 400 \mathrm{~nm}$. The ARIES retrieval of the TP is shown in Fig. 5(a). The retrieved full width at half-maximum of the intensity envelope is around $30 \mathrm{fs}$, which is consistent with the expected UV pulse duration under conditions of saturated SHG. For reference, a SEA-F-SPIDER measurement of the PP at $800 \mathrm{~nm}$ is also shown. A magnified view of the center of the waveform is shown in Fig. 5(b). The retrieved spectrum of the TP is shown in Fig. 5(c). It agrees qualitatively with an independent measurement of the UV spectrum.

For waveforms with bandwidths spanning an octave or more, the spectral response of ARIES is not uniform and needs to be taken into account. We calculated the spectral response using SFA simulations to determine the maximum cut-off shift $\left|\Delta \varepsilon_{\max }\right|$ (over all $\tau$ ) as a function of $\omega_{\text {tp }}$. The result is shown in Fig. 6 for an $800 \mathrm{~nm} \mathrm{PP}$, and TP frequencies, $\omega_{\mathrm{tp}} / 2 \pi$, in the range $0.2-2.3 \mathrm{PHz}(1500-130 \mathrm{~nm})$. The SFA result agrees well with a simulation based on numerical integration of the classical equation of motion where no assumptions are made about $t_{i}$ and $t_{r}$. Since the classical calculation does not include the field-dependent ionization dynamics, this indicates that the dominant mechanism for the cut-off shift is electron acceleration in the combined field. Reasonable agreement with the SFA result is also obtained with the classical approximation $\Delta \varepsilon_{\max } \approx m v_{\mathrm{pp}}^{(\max )} v_{\mathrm{tp}}^{(\max )}$, where $v_{\mathrm{pp}}^{(\max )}$ and $v_{\mathrm{tp}}^{(\max )}$ are the maximum recollision velocities for the PP and TP, respectively. This
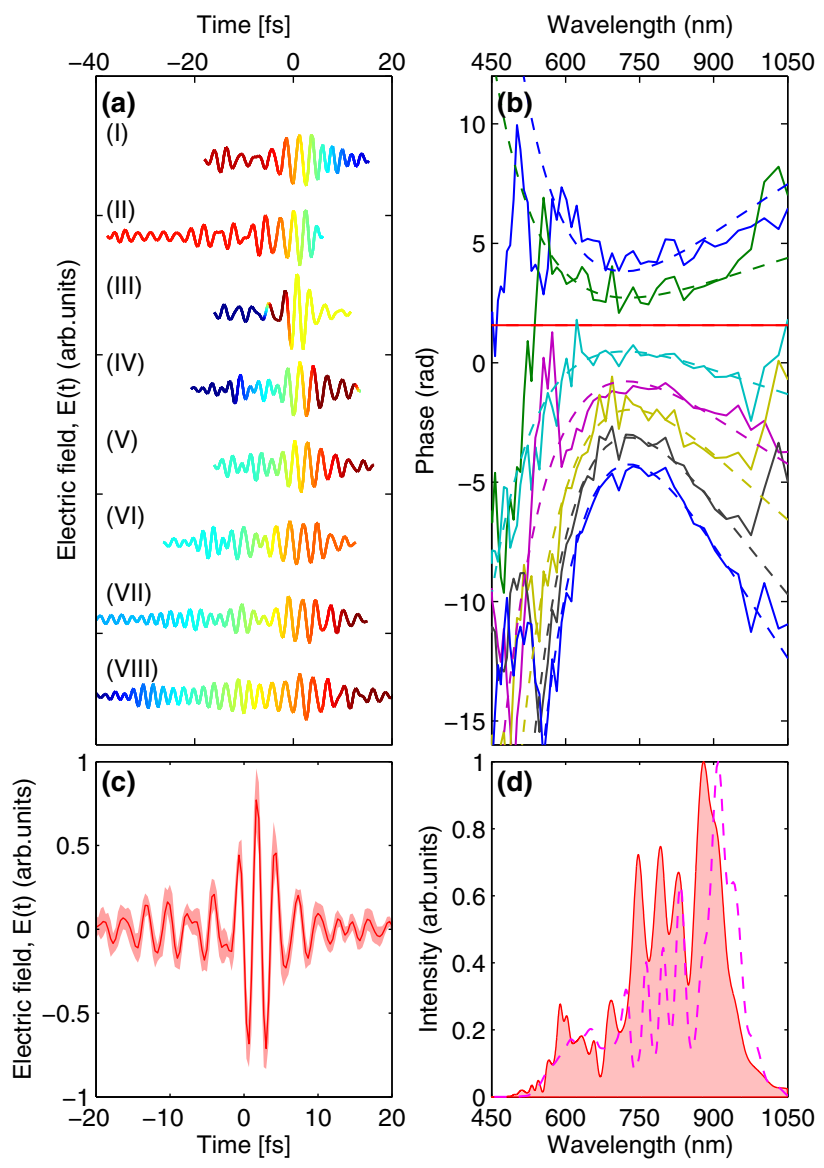

Fig. 4. (a) Retrieved waveforms for long-range TP wedge scan. The colors of the curves are related to the instantaneous frequency to show the pulse chirp (red corresponding to lower frequencies, blue to higher frequencies). The fused silica insertions relative to the zero dispersion wedge position are (I) $0.3 \mathrm{~mm}$, (II) $0.15 \mathrm{~mm}$, (III) $0 \mathrm{~mm}$, (IV) $-0.15 \mathrm{~mm}$, (V) $-0.3 \mathrm{~mm}$, (VI) $-0.45 \mathrm{~mm}$, (VII) $-0.6 \mathrm{~mm}$, and (VIII) $-0.75 \mathrm{~mm}$. (b) Spectral phase curves for the retrieved waveforms minus the spectral phase of the near-Fourier-limited pulse corresponding to (I) to (VIII), in same order as in (a). The curves have been offset for clarity. The dashed lines are the calculated phase curves from the known fused silica insertion. (c) The thin red curve is the average of the waveforms in (a), after numerical compensation of the known dispersion. The shaded region represents the mean \pm 1 standard deviation. (d) Retrieved TP spectrum from ARIES (filled red line) and TP spectrum measured using SEA-F-SPIDER (dashed magenta curve).

approximation provides a simple scaling relationship in terms of experimental parameters:

$$
\Delta \varepsilon_{\max } \approx 2 \varepsilon_{\mathrm{pp}}^{(\max )} \frac{\hat{E}_{\mathrm{tp}}}{\hat{E}_{\mathrm{pp}}} \frac{\omega_{\mathrm{pp}}}{\omega_{\mathrm{tp}}} .
$$

Though this tends to overestimate the shift compared to the SFA result, it reproduces the general features of the frequency dependence. The field sensitivity of ARIES can thus be approximated by

$$
\Delta \varepsilon_{\max } / \mathrm{eV} \approx 4 \sqrt{\varepsilon_{\mathrm{pp}}^{(\max )} / \mathrm{eV}}\left(\lambda_{\mathrm{tp}} / \mu \mathrm{m}\right)\left(\hat{E}_{\mathrm{tp}} /(\mathrm{V} / \AA)\right),
$$

where we have used the well-known result that $\varepsilon_{\mathrm{pp}}^{(\max )}=3.17 U_{p}$, where $U_{p} \propto \hat{E}_{\mathrm{pp}}^{2} / \omega_{\mathrm{pp}}^{2}$ is the ponderomotive potential. 


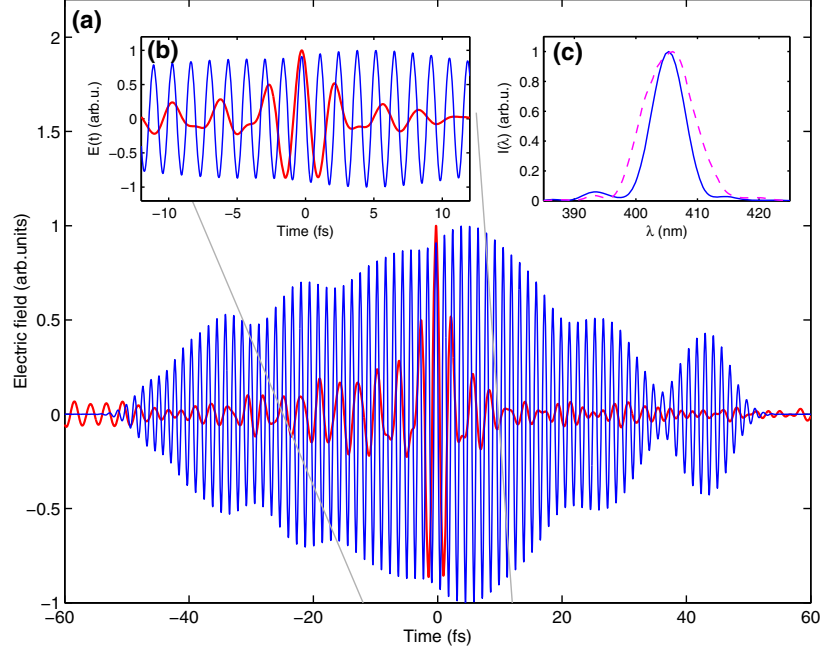

Fig. 5. ARIES measurement of a UV second-harmonic TP with a center wavelength of $\approx 400 \mathrm{~nm}$. (a) The retrieved TP waveform (blue curve) and the PP waveform measured by SPIDER (red curve). (b) Magnified view of the central portion of (a). (c) Retrieved TP spectrum (blue curve) and the TP spectrum measured by a spectrometer (magenta dashed curve).

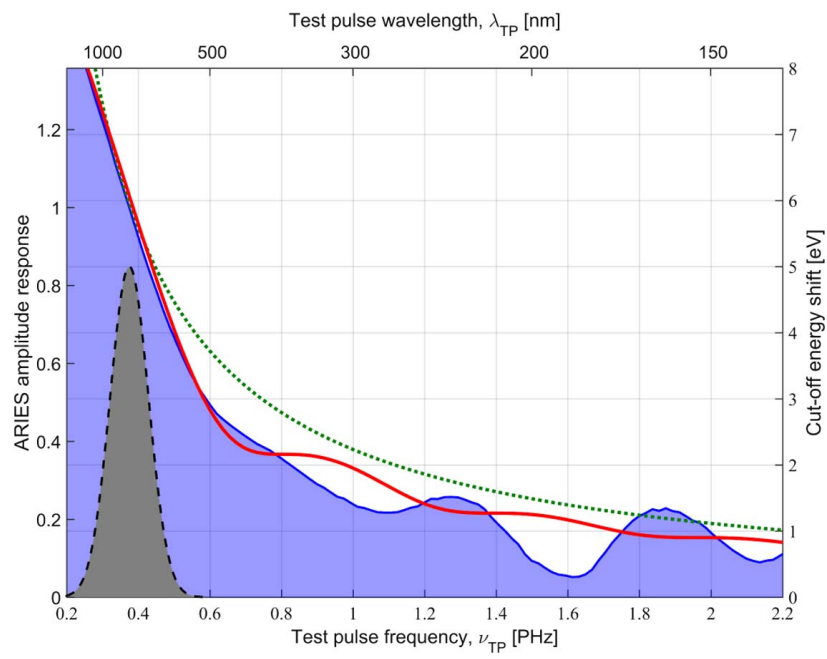

Fig. 6. ARIES amplitude response (i.e., maximum cut-off energy shift) for a $5 \times 10^{14} \mathrm{~W} \mathrm{~cm}^{-2}, 3.5 \mathrm{fs}, 800 \mathrm{~nm}$ probe pulse and CW test waveform with $I_{\mathrm{tp}} / I_{\mathrm{pp}}=0.1 \%$ as a function of the TP frequency. Filled blue curve: response calculated from SFA simulations. Red curve: response calculated from numerically integrating the classical equation of motion [Eq. (1)]. Dotted green curve: classical approximation given by [Eq. (4)]. The right vertical axis shows the absolute shift in photon energy; the left vertical axis shows the relative response scaled to unity for the SFA simulations at $\omega_{\text {tp }}=\omega_{\mathrm{pp}}$. The PP spectrum is shown by the gray-filled dashed black curve.

We now address the basic requirements for an ARIES measurement. Both the TP and PP must be CEP stabilized and synchronized with a timing jitter that is small compared to the electron excursion time, $\Delta t$. Provided the TP and PP are phase locked, CEP fluctuations are common to both, and, thus, the PP will sample the same temporal point of the TP for a given delay. Small CEP fluctuations therefore have a similar effect as intensity fluctuations by reducing the half-cycle peak intensity, resulting in a random shift of the cut-off energy quantified by a normal distribution with standard deviation

$$
\frac{\sigma_{\varepsilon_{\max }}^{(\mathrm{CEP})}}{\varepsilon_{\max }} \approx \frac{4 \ln 2}{\sqrt{N / 2}}\left(\frac{\sigma_{\mathrm{CEP}}}{\omega_{\mathrm{pP}} \tau_{\mathrm{pp}}}\right)^{2}
$$

where $\sigma_{\mathrm{CEP}}$ is the standard deviation of the CEP distribution, $\omega_{\mathrm{pp}}$ and $\tau_{\mathrm{pp}}$ are the PP central frequency and full-width at halfmaximum pulse duration, respectively, and the measured HHG spectrum is averaged over $N$ laser pulses. For our experimental parameters $\left(\sigma_{\mathrm{CEP}} \approx 250 \mathrm{mrad}, \quad N \approx 300, \omega_{\mathrm{pp}}=2.36 \mathrm{rad} / \mathrm{fs}\right.$, $\tau_{\mathrm{pp}}=3.5 \mathrm{fs}, \varepsilon_{\max } \approx 100 \mathrm{eV}$ ), we estimate the noise amplitude in the reconstructed field due to CEP fluctuations to be $\approx 0.02 \mathrm{eV}$, which is $\approx 0.7 \%$ of the $3 \mathrm{eV}$ maximum shift due to the TP. Though negligible in our experiment, this can be reduced by numerically bandpass filtering the reconstructed field in the spectral domain because the CEP noise is uncorrelated with the TP-PP delay.

We now consider the pulse duration requirements. Though we used a few-cycle PP in our experiments, our SFA simulations suggest that this is not essential, though further experiments are required to confirm this. Figure 7 shows the simulated ARIES retrievals for 4 and 24 fs PPs. The retrieval using the $24 \mathrm{fs} \mathrm{PP}$ is still satisfactory. Since the HHG cut-off emission is generated from the most intense half-cycle of the PP field [37], a welldefined gate for the measurement (between the ionization and recollision times for the cut-off emission) is obtained even for a multicycle PP. The reconstruction error can be quantified by calculating the RMS field error [38] between the ARIES reconstructed TP field and the TP field used in the simulation. It increases relatively slowly with TP duration up to about $15 \mathrm{fs}$. The reconstruction error at $24 \mathrm{fs}$ is approximately double that at $4 \mathrm{fs}$.
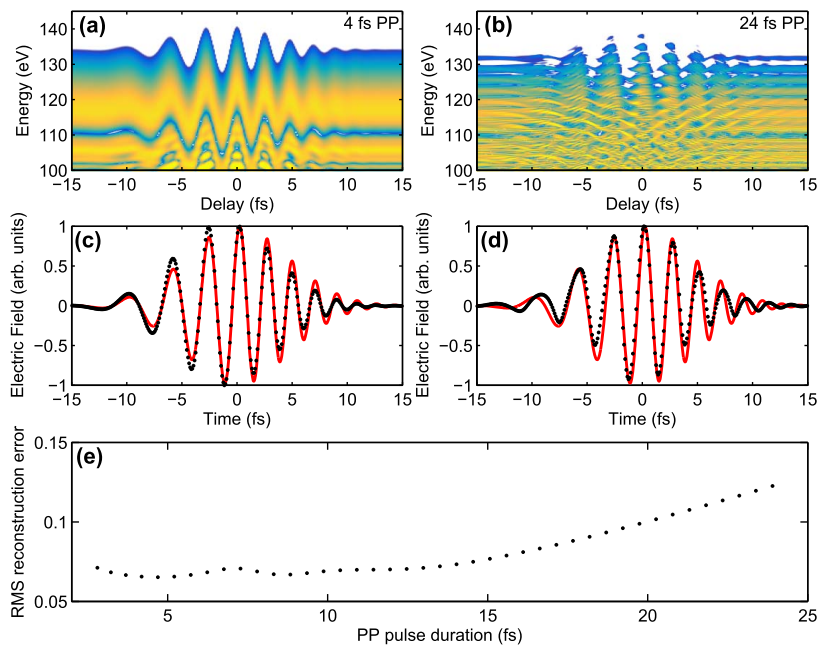

Fig. 7. SFA simulations of ARIES using multicycle PPs. The PPs are centered at wavelength of $800 \mathrm{~nm}$ with peak intensity fixed at $5 \times 10^{14} \mathrm{~W} \mathrm{~cm}^{-2}$. The positively chirped TP is $7.7 \mathrm{fs}$ in duration and centered at $800 \mathrm{~nm}$, with peak intensity $0.1 \%$ of the PP. ARIES traces for (a) a $4 \mathrm{fs}$ and (b) a $24 \mathrm{fs}$ PP. (c),(d) ARIES reconstructions of the TP electric field (black points) compared to the TP field used in the calculation (red lines) corresponding to (a) and (b), respectively. (e) ARIES reconstruction error as a function of PP pulse duration (see text for details). 
In terms of the intensity requirements, the PP must be sufficiently intense to drive HHG in the nonperturbative regime (typically $I_{\mathrm{pp}} \gtrsim 10^{14} \mathrm{Wcm}^{-2}$ ), and the TP must be intense enough to cause a measurable cut-off shift, but weak enough to ensure a linear relationship between the cut-off shift and the electric field. For our experimental conditions, our SFA simulations indicate that the linearity error is $<5 \%$ for $I_{\mathrm{tp}} / I_{\mathrm{pp}}<1 \%$. Intensity fluctuations add a small amount of noise to the reconstructed electric field. Since the random intensity fluctuations are normally distributed with a standard deviation $\sigma_{I}$, the cut-off energy will also be normally distributed with standard deviation $\sigma_{\varepsilon_{\max }}^{(I)} / \varepsilon_{\max } \approx \frac{1}{\sqrt{N}} \sigma_{I} / I$, where the HHG spectrum has been averaged over $N$ laser pulses. For our experimental parameters $\left(\sigma_{I} / I \approx 2 \%, N \approx 300, \varepsilon_{\max } \approx 100 \mathrm{eV}\right)$, we estimate the noise amplitude on the reconstructed field due to intensity fluctuations to be $\approx 4 \%$. As was the case for the CEP noise, this can be reduced by bandpass filtering the reconstructed field in the spectral domain.

How strong a TP field is required for an ARIES measurement? In our experiment, using a relatively low-resolution spectrometer, we were able to resolve a cut-off shift of $\approx 3 \mathrm{eV}$ at a photon energy of $100 \mathrm{eV}$, which is equivalent to resolving neighboring harmonics of an $800 \mathrm{~nm}$ laser. Taking this as a conservative value for the available spectral resolution, then from Eq. (5) with $\lambda_{\text {tp }}=$ $800 \mathrm{~nm}$, a minimum TP field of $\gtrsim 0.1 \mathrm{~V} / \AA$ is required, i.e., $I_{\text {tp }} \gtrsim 1.3 \times 10^{11} \mathrm{~W} \mathrm{~cm}^{-2}$. For typical HHG focusing conditions (focused spot radius of $\approx 50 \mu \mathrm{m}$ ), this intensity could be reached with a TP energy of $\approx 0.25 \mu \mathrm{J}$ for a 50 fs pulse duration, or proportionally less for a shorter TP. Therefore, the electric field of TPs at the $100 \mathrm{~nJ}$ level should be retrievable using ARIES.

For situations where the TP will be used for HHG, such as tailored fields for optimized XUV generation or attosecond pulse generation, ARIES provides an in situ measurement that can be carried out with the spectrometers already found on most HHG beamlines. Implementation is greatly facilitated by the use of collinear beams, which make alignment simple and robust. The collinear geometry is also compatible with HHG gas targets that have small limiting apertures. These include commonly used pulsed and continuously flowing drilled-tube targets, as well as gas-filled waveguides that are used to improved phase matching $[39,40]$ or to extend the HHG interaction region for tightly focused beams, as required when using very low laser pulse energies [41,42].

For our proof-of-concept measurements, we used about 2000 delay points for each ARIES scan, typically oversampling the waveform by about 10 times the Nyquist rate. Each scan took about 10 min to complete, since we integrated the HHG spectrum at each delay position over $\approx 300$ laser pulses $(\approx 300 \mathrm{~ms}$ at $1 \mathrm{kHz}$ laser repetition rate) to increase the signal-to-noise ratio for the detection of the relatively high cut-off photon energies we used $(>100 \mathrm{eV})$. In principle, the ARIES scan time could be reduced to a few seconds for a $1 \mathrm{kHz}$ source (or less for higher repetition rate lasers), by reducing the sampling rate to closer to the Nyquist rate and significantly decreasing the integration period, e.g., by using a lower photon energy cutoff where both the HHG signal and detection efficiency are higher. Since the retrieval is computationally straightforward, ARIES thus lends itself well to (near-) real-time monitoring of optical waveforms. For example, one could imagine ARIES being used to provide a feedback signal for a closed-loop waveform synthesizer $[17,18]$ to allow the rapid convergence to a target waveform, or to provide a measurement of the excitation light field in a coherent control experiment [43].

Due to the close agreement between the classical and SFA simulations, we conclude that the main mechanism for modulating the harmonic spectrum is from steering the electron trajectory in the continuum. Therefore, we expect ARIES can be used in a variety of configurations beyond those explicitly demonstrated here. For example, we believe it is possible to use alternative gating schemes, such as polarization gating, although this has yet to be demonstrated. ARIES is not limited to neon or a cutoff at $120 \mathrm{eV}$. For example, we have successfully demonstrated ARIES in argon with a cutoff at $60 \mathrm{eV}$. Using a cutoff at higher energies has the advantage of decreasing the linearity error in the reconstructed electric field due to reducing the effect of the ionization potential and the terms dropped in Eq. (4). We also believe ARIES can be used at different PP wavelengths. However, shorter PP wavelengths might decrease the accuracy due to increased multiphoton ionization effects and the breakdown of the SFA; conversely, increasing the PP wavelength should improve the accuracy.

\section{CONCLUSIONS}

In summary, we have theoretically and experimentally demonstrated a new all-optical technique, ARIES, based on HHG for measuring the time-dependent field of ultrafast optical waveforms. We used ARIES to retrieve the electric field of femtosecond pulses centered at 800 and $400 \mathrm{~nm}$. ARIES uses collinear beams, which greatly simplifies implementation and analysis, and allows the technique to be applied to a range of HHG targets, such as gas-filled waveguides, where crossed beams would be problematic. Since the ARIES signal is the delay-dependent shift of the HHG cutoff, no spatial resolution is required in the measurement of the HHG spectrum. With retrieval rates approaching $\approx 1 \mathrm{~Hz}$ possible with a $1 \mathrm{kHz}$ repetition-rate source, real-time measurement and optimization of complex optical waveforms is within reach. See [44] for supporting content.

Funding. Engineering and Physical Sciences Research Council (EPSRC) (EP/F034601/1, EP/I032517/1); European Research Council (ERC) (ASTEX project 290467); European Commission (Marie Curie ITN EC317232).

Acknowledgment. The authors thank Dane Austin for useful discussions and Peter Ruthven and Andy Gregory for technical support.

\section{REFERENCES}

1. E. Potter, J. Herek, S. Pedersen, Q. Liu, and A. Zewail, "Femtosecond laser control of a chemical reaction," Nature 355, 66-68 (1992).

2. P. Brumer and M. Shapiro, "Control of unimolecular reactions using coherent light," Chem. Phys. Lett. 126, 541-546 (1986).

3. A. Assion, T. Baumert, M. Bergt, T. Brixner, B. Kiefer, V. Seyfried, M. Strehle, and G. Gerber, "Control of chemical reactions by feedbackoptimized phase-shaped femtosecond laser pulses," Science $\mathbf{2 8 2}$, 919-922 (1998).

4. R. J. Levis, G. M. Menkir, and H. Rabitz, "Selective bond dissociation and rearrangement with optimally tailored, strong-field laser pulses," Science 292, 709-713 (2001). 
5. F. Krausz and M. Ivanov, "Attosecond physics," Rev. Mod. Phys. 81, 163 (2009).

6. A. Y. Vorobyev and C. Guo, "Direct femtosecond laser surface nano/ microstructuring and its applications," Laser Photon. Rev. 7, 385-407 (2013).

7. C.-S. Yang, C.-H. Lin, A. Zaytsev, K.-C. Teng, T.-H. Her, and C.-L. Pan, "Femtosecond laser ablation of polymethylmethacrylate via dual-color synthesized waveform,” Appl. Phys. Lett. 106, 051902 (2015).

8. L. Xu, T. Hänsch, C. Spielmann, A. Poppe, T. Brabec, and F. Krausz, "Route to phase control of ultrashort light pulses," Opt. Lett. 21, 2008-2010 (1996).

9. A. Baltuška, T. Udem, M. Uiberacker, M. Hentschel, E. Goulielmakis, Ch. Gohle, R. Holzwarth, V. S. Yakovlev, A. Scrinzi, T. W. Hänsch, and F. Krausz, "Attosecond control of electronic processes by intense light fields," Nature 421, 611-615 (2003).

10. I. A. Walmsley and C. Dorrer, "Characterization of ultrashort electromagnetic pulses," Adv. Opt. Photon. 1, 308-437 (2009)

11. R. Trebino, K. W. DeLong, D. N. Fittinghoff, J. N. Sweetser, M. A. Krumbügel, B. A. Richman, and D. J. Kane, "Measuring ultrashort laser pulses in the time-frequency domain using frequency-resolved optical gating," Rev. Sci. Instrum. 68, 3277-3295 (1997).

12. C. laconis and I. A. Walmsley, "Spectral phase interferometry for direct electric-field reconstruction of ultrashort optical pulses," Opt. Lett. 23, 792-794 (1998).

13. V. V. Lozovoy, I. Pastirk, and M. Dantus, "Multiphoton intrapulse interference. IV. Ultrashort laser pulse spectral phase characterization and compensation," Opt. Lett. 29, 775-777 (2004).

14. M. Miranda, C. L. Arnold, T. Fordell, F. Silva, B. Alonso, R. Weigand, A. L'Huillier, and H. Crespo, "Characterization of broadband few-cycle laser pulses with the D-scan technique," Opt. Express 20, 18732-18743 (2012).

15. D. Keusters, H.-S. Tan, P. O'Shea, E. Zeek, R. Trebino, and W. S. Warren, "Relative-phase ambiguities in measurements of ultrashort pulses with well-separated multiple frequency components," J. Opt. Soc. Am. B 20, 2226-2237 (2003).

16. D. R. Austin, T. Witting, and I. A. Walmsley, "Resolution of the relative phase ambiguity in spectral shearing interferometry of ultrashort pulses," Opt. Lett. 35, 1971-1973 (2010).

17. A. Wirth, M. Th. Hassan, I. Grguraš, J. Gagnon, A. Moulet, T. T. Luu, S. Pabst, R. Santra, Z. A. Alahmed, A. M. Azzeer, V. S. Yakovlev, V. Pervak, F. Krausz, and E. Goulielmakis, "Synthesized light transients," Science 334, 195-200 (2011).

18. O. Mucke, S. Fang, G. Cirmi, G. M. Rossi, S.-H. Chia, H. Ye, Y. Yang, R. Mainz, C. Manzoni, P. Farinello, G. Cerullo, and F. X. Kartner, "Toward waveform nonlinear optics using multimillijoule sub-cycle waveform synthesizers," IEEE J. Sel. Top. Quantum Electron. 21, 8700712 (2015).

19. H.-S. Chan, Z.-M. Hsieh, W.-H. Liang, A. Kung, C.-K. Lee, C.-J. Lai, R.-P. Pan, and L.-H. Peng, "Synthesis and measurement of ultrafast waveforms from five discrete optical harmonics," Science 331, 1165-1168 (2011).

20. T. Luu, M. Garg, S. Y. Kruchinin, A. Moulet, M. T. Hassan, and E. Goulielmakis, "Extreme ultraviolet high-harmonic spectroscopy of solids," Nature 521, 498-502 (2015).

21. L. Chipperfield, J. Robinson, J. Tisch, and J. Marangos, "Ideal waveform to generate the maximum possible electron recollision energy for any given oscillation period," Phys. Rev. Lett. 102, 063003 (2009).

22. S. Haessler, T. Balčiunas, G. Fan, G. Andriukaitis, A. Pugžlys, A Baltuška, T. Witting, R. Squibb, A. Zaïr, J. W. G. Tisch, J. P. Marangos, and L. E. Chipperfield, "Optimization of quantum trajectories driven by strong-field waveforms," Phys. Rev. X 4, 021028 (2014).

23. P. G. de Alaiza Martínez, I. Babushkin, L. Bergé, S. Skupin, E. CabreraGranado, C. Köhler, U. Morgner, A. Husakou, and J. Herrmann, "Boosting terahertz generation in laser-field ionized gases using a sawtooth wave shape," Phys. Rev. Lett. 114, 183901 (2015).

24. J. Itatani, F. Quéré, G. L. Yudin, M. Y. Ivanov, F. Krausz, and P. B. Corkum, "Attosecond streak camera," Phys. Rev. Lett. 88, 173903 (2002).
25. Y. Mairesse and F. Quéré, "Frequency-resolved optical gating for complete reconstruction of attosecond bursts," Phys. Rev. A 71, 011401 (2005).

26. E. Goulielmakis, M. Uiberacker, R. Kienberger, A. Baltuska, V. Yakovlev, A. Scrinzi, Th. Westerwalbesloh, U. Kleineberg, U. Heinzmann, M. Drescher, and F. Krausz, "Direct measurement of light waves," Science 305, 1267-1269 (2004).

27. T. Witting, F. Frank, W. Okell, C. Arrell, J. Marangos, and J. Tisch, "Sub-4-fs laser pulse characterization by spatially resolved spectral shearing interferometry and attosecond streaking," J. Phys. B 45, 074014 (2012)

28. M. T. Hassan, A. Wirth, I. Grguraš, A. Moulet, T. T. Luu, J. Gagnon, V. Pervak, and E. Goulielmakis, "Attosecond photonics: synthesis and control of light transients," Rev. Sci. Instrum. 83, 111301 (2012).

29. K. T. Kim, C. Zhang, A. D. Shiner, B. E. Schmidt, F. Légaré, D. Villeneuve, and P. Corkum, "Petahertz optical oscilloscope," Nat. Photonics 7, 958-962 (2013).

30. P. B. Corkum, "Plasma perspective on strong field multiphoton ionization," Phys. Rev. Lett. 71, 1994-1997 (1993).

31. T. Pfeifer, L. Gallmann, M. J. Abel, P. M. Nagel, D. M. Neumark, and S. R. Leone, "Heterodyne mixing of laser fields for temporal gating of highorder harmonic generation," Phys. Rev. Lett. 97, 163901 (2006).

32. M. Lewenstein, P. Balcou, M. Y. Ivanov, A. L'Huillier, and P. B. Corkum, "Theory of high-harmonic generation by low-frequency laser fields," Phys. Rev. A 49, 2117-2132 (1994).

33. F. Frank, C. Arrell, T. Witting, W. A. Okell, J. McKenna, J. S. Robinson, C. A. Haworth, D. Austin, H. Teng, I. A. Walmsley, J. P. Marangos, and J. W. G. Tisch, "Invited review article: technology for attosecond science," Rev. Sci. Instrum. 83, 071101 (2012).

34. T. Witting, F. Frank, C. A. Arrell, W. A. Okell, J. P. Marangos, and J. W. Tisch, "Characterization of high-intensity sub-4-fs laser pulses using spatially encoded spectral shearing interferometry," Opt. Lett. 36, 1680-1682 (2011).

35. T. Witting, D. R. Austin, and I. A. Walmsley, "Improved ancilla preparation in spectral shearing interferometry for accurate ultrafast pulse characterization," Opt. Lett. 34, 881-883 (2009).

36. N. Nakano, H. Kuroda, T. Kita, and T. Harada, "Development of a flatfield grazing-incidence XUV spectrometer and its application in picosecond XUV spectroscopy," Appl. Opt. 23, 2386-2392 (1984).

37. C. A. Haworth, L. E. Chipperfield, J. S. Robinson, P. L. Knight, J. P. Marangos, and J. W. Tisch, "Half-cycle cutoffs in harmonic spectra and robust carrier-envelope phase retrieval," Nat. Phys. 3, 52-57 (2007).

38. C. Dorrer and I. A. Walmsley, "Accuracy criterion for ultrashort pulse characterization techniques: application to spectral phase interferometry for direct electric field reconstruction," J. Opt. Soc. Am. B 19, 1019-1029 (2002).

39. A. Rundquist, C. G. Durfee, Z. Chang, C. Herne, S. Backus, M. M. Murnane, and H. C. Kapteyn, "Phase-matched generation of coherent soft x-rays," Science 280, 1412-1415 (1998).

40. N. H. Shon, A. Suda, Y. Tamaki, and K. Midorikawa, "High-order harmonic and attosecond pulse generations: bulk media versus hollow waveguides," Phys. Rev. A 63, 063806 (2001)

41. O. H. Heckl, C. R. E. Baer, C. Kränkel, S. V. Marchese, F. Schapper, M. Holler, T. Südmeyer, J. S. Robinson, J. W. G. Tisch, F. Couny, P. Light, F. Benabid, and U. Keller, "High harmonic generation in a gas-filled hollow-core photonic crystal fiber," Appl. Phys. B 97, 369-373 (2009).

42. J. C. Travers, W. Chang, J. Nold, N. Y. Joly, and P. St. J. Russell, "Ultrafast nonlinear optics in gas-filled hollow-core photonic crystal fibers," J. Opt. Soc. Am. B 28, A11-A26 (2011).

43. J. L. Herek, W. Wohlleben, R. J. Cogdell, D. Zeidler, and M. Motzkus, "Quantum control of energy flow in light harvesting," Nature 417, 533-535 (2002)

44. http://dx.doi.org/10.5287/bodleian:pv7DopPz7. 